\title{
The Impact of Interactivity on Virtual Gifts Giving Intent —-Based on Live-Streaming Platforms
}

\author{
Jun Fan ${ }^{1, a}$,Qing Zhang ${ }^{2, b}$ \\ ${ }^{1}$ School of Business Administration, Zhejiang Gongshang University, Hangzhou, Zhejiang, \\ China \\ ${ }^{2}$ School of Business Administration, Zhejiang Gongshang University, Hangzhou, Zhejiang, \\ China \\ afj@zjgsu.edu.cn, b1183691204@qq.com \\ ${ }^{*}$ Qing Zhang
}

Keywords: Live-Streaming, Interactive, Type, Interactive, Strategy, Emotional, Energy, Broadcaster Identification.

\begin{abstract}
Using interaction ritual theory and social identity theory, this research examines how the interactive types and interactive strategies in live-streaming under two different live-streaming types influence the audience's gifts giving intent will through a $2 \times 2 \times 2$ experimental designs. The results shows that: (1) When using task-oriented interaction and the updog strategy in the video games live-streaming can arouse more emotional energy and broadcaster identification of the audience. (2)When using relation-oriented interaction and the underdog strategy in the talent shows live-streaming can arouse more emotional energy and broadcaster identification of the audience.(3) The emotional energy has a significant positive influence on broadcaster identification and the virtual gifts giving intent,and the virtual gifts giving intent is also influenced by the broadcaster identification significantly.
\end{abstract}

\section{不同直播类型下主播互动类型和互动策略 对受众打赏意愿的影响 \\ 范钧 $1, a$, 张情 $2, \mathrm{~b}$ \\ 1 浙江工商大学工商管理学院, 杭州, 浙江, 中国 \\ 2 浙江工商大学工商管理学院, 杭州, 浙江, 中国 \\ Afj@zjgsu.edu.cn, b1183691204@qq.com \\ *张情}

关键词：网络直播、互动类型、互动策略、情感能量、主播认同、打赏意愿

中文摘要.运用互动仪式链理论和社会认同理论, 并通过一个 $2 \times 2 \times 2$ 实验, 分析了不同直播类 型、互动类型和互动策略下, 游戏直播和秀场直播中, 不同互动类型和互动策略对受众打赏 意愿的影响。研究表明:（1）游戏直播中, 采取任务导向型互动及示强策略时, 受众的情感 能量和主播认同相对较强; （2）秀场直播中, 采取关系导向型互动及示弱策略时, 受众的情 感能量和主播认同相对较强; （3）情感能量对主播认同和打赏意愿有显著正向影响，主播认 同对受众打赏意愿有显著正向影响。 


\section{1. 引言}

随着人们生活水平的不断提高, 消费者的消费结构不断升级, 使得网络直播行业在这样 的消费结构升级中得以迅速成长。网络直播的主要收入是用户充值后购买虚拟道具, 即来自 用户的打赏。值得注意的是，自 2016 年直播超级大风口，两年年多的时间，从蓝海烧成了红 海，普遍亏损、盈利艰难、现金流紧张等关键词成为中小直播平台的速写标签。目前直播平 台的盈利模式主要是靠用户的打赏，因此，要想持续的运作，如何激发用户的打赏意愿是网 络直播平台需要考虑的问题。网络直播最主要的一个要素就是互动, 研究互动对打赏意愿的 影响是有实践基础和理论基础的, 互动是本文研究的主线。

首先，直播的种类会影响受众的行为意向，已有研究显示视频的种类可以影响受众的观 看、分享和评论等行为 (Khan\&Vong)。据此, 直播的种类也会影响用户的行为意向, 当前, 大多数直播平台主要是根据直播的内容来划分直播频道的, 最流行的直播种类就是游戏直播 和秀场直播 ( Hu M et al.,2017)。

其次, 从互动的角度看, 游戏直播和秀场直播在主播互动风格上面是有差异的, 游戏直 播中受众主要是获取知识和学习游戏技能, 因此认为游戏主要是侧重任务互动, 而秀场直播 中受众主要目的是释放压力, 主要侧重娱乐和社交, 因此秀场直播主要是侧重关系互动, 这 是基于 (Kohler et al.,2011)互动类型的划分方法, 将互动类型划分为任务导向型和关系导向型 两种。

再次，从消费者自身的特质来看，将主播的互动策略划分为示强和示弱策略，消费者有 时候更愿意去支持弱者, 人们参与直播的很大一部分原因在于他们在现实中找不到存在感, 只有在直播间里面感受到自己对主播有一种控制的感觉, 如果主播表现的比消费者还要劣势, 这时会产生劣势者效应，消费者会对主播产生一种同情的感觉，从而促进受众的打赏。

\section{2. 理论基础和研究假设}

\section{1 理论基础}

\section{1 .1 互动仪式链理论}

Collins（2004）的互动仪式链理论来研究互动对打赏意愿的影响，网络直播满足互动仪 式产生的四个要素, 首先主播和受众基于共同的兴趣爱好聚集在同一个直播平台和同一个房 间, 满足了同时在场这一要素; 受众通过发送弹幕、点赞、关注等和主播对话，并以赠送平 台的虚拟礼物的形式进行互动，对于直播间以外的人设定了界限; 受众在进行互动交流的过 程中，都拥有共同的关注焦点，在游戏直播中，如“游戏操作、主播、共同的情感”等。随着 受众之间的互动行为的进行，产生情感的刺激“主播的行为、游戏的操作结果”，这种情感刺 激会产生情感能量, 情感能量是互动仪式产生的直接驱动力(Baker,2010), 打赏实质上是一种 受众与主播的互动行为, 通过主播的互动受众获得了情感能量, 从而促进了打赏。

\subsection{2 劣势者效应和社会认同理论}

劣势者效应(underdog effect)一词主要是出现在竞争的场合中，参与竞争的企业往往都不 愿成为劣势的一方，总是乐于“示强”，差于“示弱”。然而有时候劣势者勇于承认并展示自己 的弱势，反而可以赢得获胜的机会，劣势者效应发生的中介之一便是社会认同机制; Paharia 等（2011）针对消费者的多项实验表明，消费者对劣势品牌表现出更高的购买意愿和品牌忠 诚度。这种示弱营销策略与传统的“示强“策略明显不同。据此，本文将主播对受众的互动策 略分为示强和示弱策略, 受众如果觉得主播相对于自己来说是劣势者, 可能就会增强对主播 的打赏意愿。因此主播对受众示弱，会增强打赏意愿吗？示强一定不适用吗？不同的直播类 型会存在不一样的结果吗? 这都是要考虑的问题。此外, 对于不同的互动类型是否适用于不 
同的互动策略，也是本文深入探讨的问题。

\section{2 研究假设}

2.2.1 不同直播类型，不同互动类型时，互动策略对受众情感能量的影响

首先, 游戏直播侧重任务导向型互动, 即侧重游戏任务本身, 即游戏; 而秀场直播更加 侧重关系导向型互动, 侧重受众的情感和社会需求。

其次, 本文从观看直播的实践中以及从文献中得出, 主播的示强和示弱的互动策略对受 众的情感能量也是有影响的。在游戏直播中, 受众主要是期待看到主播技术厉害的一面, 这 也是为什么直播平台界面上显示如: “国服第一马克”、“Dv 大哥电灯，不服来恐”、“这个公 孙离有点小秀“等的原因, 标题就彰显出主播的过人之处, 更能激发受众的兴趣。因此主播在 以任务导向型互动为主体时, 主播要表现出自己游戏厉害的一面, 实际操作可能没有那么出 色，但是在游戏解说上一定要给受众信心，在用户面前“示强”，从而激发受众的情感能量。

在游戏直播中，在关系导向型互动时，主播应该在受众面前“示弱”。有的游戏主播虽然 游戏技术不是很好, 但是在和受众的互动策略中表现出很大的优势。在直播中, 如果受众感 受到主播在和自己”示弱“，如主播和自己诉说自己的难处、卖萌等，都是在“示弱”，这时会 产生劣势者效应, 受众会对主播有一种同情和想要帮助的感觉, 从而激发受众的情感能量。 因此，本文提出如下假设。

$\mathrm{H} 1$ : 不同直播类型下, 不同的互动类型时, 主播互动策略对受众情感能量的影响存在差 异。

H1a:游戏直播中，在任务导向型互动下，主播的示强策略相比于示弱策略更易增强受众 的情感能量。

$\mathrm{H} 1 \mathrm{~b}$ : 游戏直播中, 在关系导向型互动下, 主播的示弱策略相比于示强策略更易增强受众 的情感能量。

H1c:秀场直播中, 在任务导向型互动下, 主播的示强策略相比于示弱策略更易增强受众 的情感能量。

$\mathrm{H} 1 \mathrm{~d}$ : 秀场直播中, 游戏直播中, 在关系导向型互动下, 主播的示弱策略相比于示强策略 更易增强受众的情感能量。

\subsection{2 不同直播类型，不同互动策略时，互动类型对受众主播认同的影响}

认同是一种接受与认可的感觉, Kelman（1961）认为认同的过程就是追求与他人相似的 过程。据此, 在网络直播中以人体认同为基础的便表现为主播认同, 主播认同就是受众与主 播之间建立起密切关系并形成的归属感。与情感能量的假设基本一致, 因此, 本文提出如下 假设。

$\mathrm{H} 2$ : 不同直播类型下, 不同的互动类型时, 主播互动策略对受众主播认同的影响存在差 异。

$\mathrm{H} 2 \mathrm{a}:$ 游戏直播中, 在任务导向型互动下, 主播的示强策略相比于示弱策略更易增强受众 的主播认同。

$\mathrm{H} 2 \mathrm{~b}$ : 游戏直播中, 在关系导向型互动下, 主播的示弱策略相比于示强策略更易增强受众 的主播认同。

$\mathrm{H} 2 \mathrm{c}$ : 秀场直播中, 在任务导向型互动下, 主播的示强策略相比于示弱策略更易增强受众 的主播认同。

$\mathrm{H} 2 \mathrm{~d}$ : 秀场直播中, 在关系导向型互动下, 主播的示弱策略相比于示强策略更易增强受众 的主播认同。

2.2.3 情感能量、主播认同与受众打赏意愿的关系 
（1）情感能量对受众主播认同的影响。情感能量它并非通常意义上的“情感”,但情感能量 和情感密不可分, 受众在观看直播过程中, 对主播的互动产生情感能量时, 会对互动充满热 情, 此时就会趋向于接受主播传授的信息, 并促进其对主播的认同, 因此, 本文提出如下假 设:

H3：情感能量正向影响受众的主播认同

（2）情感能量和主播认同对受众打赏意愿的影响。情感能量驱使受众与主播互动，因此 当用户情感能量最大化时, 用户便可能会打赏主播, 因此情感能量会对用户打赏意愿产生积 极促进作用。与此同时, 受众可能因为认同主播, 欣赏他们的游戏技能, 欣赏他们的歌声等, 所以通过打赏的方式表达欣赏, 这个层面决定了受众是否打赏, 因此, 本文提出如下假设:

$\mathrm{H} 4$ :情感能量正向影响受众打赏意愿。

$\mathrm{H} 5$ :主播认同正向影响受众打赏意愿。

综上所述, 本研究的概念模型见图 1 。

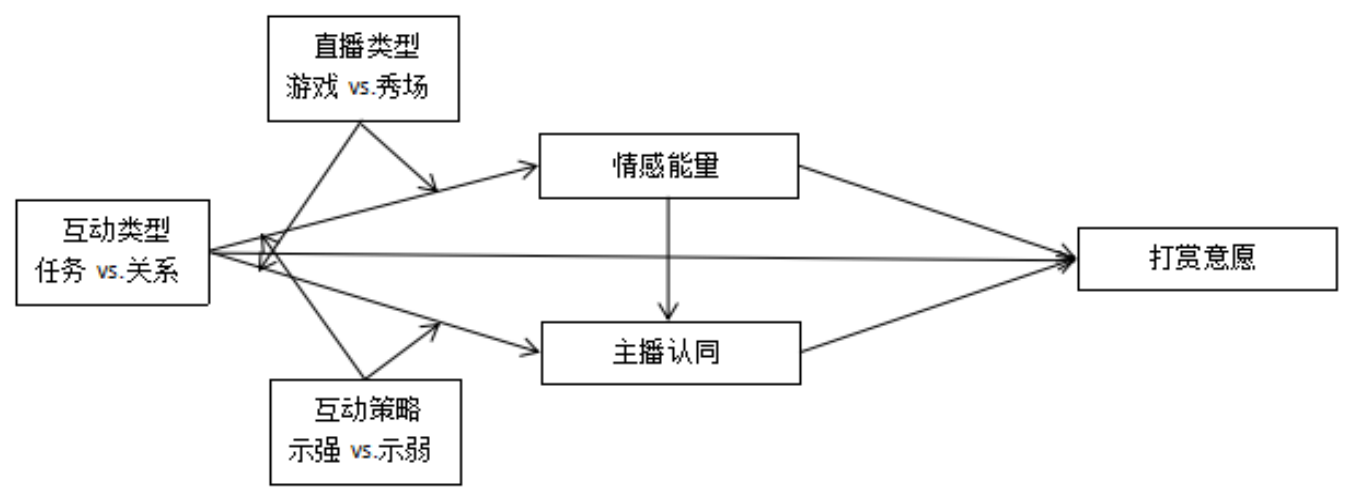

图 1 本研究的概念模型

\section{3. 研究方法}

\section{1 实验材料准备}

在直播视频的选取上，分别在游戏直播中选取视频 1 任务示强、视频 1 任务示弱、1 关 系示强、 1 关系示弱、秀场直播中选取 2 任务示强、视频 2 任务示弱、 2 关系示强、 2 关系示 弱、根据任务-关系和示强-示弱契合度量表以及单因素方差分析, 得出这 8 组视频互动类型 和互动策略操控成功, 可以作为正式实验的材料。

\section{2 正式实验}

实验采用 2 (直播类型: 游戏 / 秀场 $) \times 2$ (互动类型: 任务导向型 / 关系导向型 $) \times 2 （$ 互 动策略: 示强 / 示弱) 的组间实验设计。通过前面的预测试, 本研究从王者荣耀直播和唱歌 直播中, 分别选取了 4 个备选视频。因此总共有 8 个视频。320 名被试参与了本次实验, 并 被随机分成 8 组（每组 40 人）。在网络比较流畅的前提下, 我们用手机分别向各组发送相应 的视频, 并要求被试在观看完视频后完成相应的问卷。实验共发出 320 份问卷, 回收 280 份 问卷，剔除无效问卷后，共得到 260 份有效问卷。

\section{3 变量测量}

本研究问卷采用李克特 5 点量表形式。对于前测的问卷主要是对视频刺激物的笁选, 内 容主要是互动类型和互动策略, 互动类型主要参考了 Kohler 等 (2011) 的量表, 从任务导向 互动和关系导向互动两个维度进行测量;互动策略主要参考了 Paharia 等 (2011) 的“劣势者量 表”, 参照劣势者量表的其中一个维度, 即外部劣势 (external disadvatage)写出示弱策略的题 
项, 再根据示弱策略写出示强策略的反向量表。后期正式实验的问卷内容包括情感能量、主 播认同、打赏意愿及个人基本信息等。情感能量主要参考了 $\operatorname{Shirom}(2003)$ 的量表, 原始量表 是对同事和客户的情感能量, 共有四个题项, 本文做了适当的调整; 主播认同的测量主要参 考了 Shamir 等(1998)的量表; 打赏意愿主要参考购买意愿的量表, 主要参考了 Dodds 等(1991) 的量表。

\section{4. 数据分析和结论}

\section{1 假设检验和结论}

在正式检验假设前, 需要检验问卷的信度和效度, 本研究的问卷数据整体的 a 系数达到 了 $0.863, \mathrm{KMO}$ 系数为 0.702 , 因子载荷为 0.888 , 因此量表具有较好的信度和效度。首先验 证不同直播类型下, 不同的互动类型时, 主播互动策略对受众情感能量的影响是否存在差异。 见表 1 和图 2、表 2 和图 3。可以看出游戏直播中不论是任务还是关系导向型互动, 都是采 取示强策略比采取示弱策略效果更好。不论是采取示强还是示弱策略, 任务导向型互动都比 关系导向型的互动效果要好。结果支持假设 $\mathrm{H} 1 \mathrm{a}$,但是不支持假设 $\mathrm{H} 1 \mathrm{~b}$ 。即：在游戏直播中, 不管是怎样的互动类型, 示强策略都优于示弱策略, 不论怎样的互动策略, 都是任务导向型 互动优于关系导向型互动。

表 1 游戏直播中不同互动类型、互动策略对情感能量的影响

\begin{tabular}{|l|l|l|l|l|l|}
\hline & 因变量 & 自由度 & 均方 & F统计量 & P值 \\
\hline \multirow{5}{*}{ 情感能量 } & 互动类型 & 15 & 0.317 & 1.309 & 0.211 \\
\cline { 2 - 6 } & 互动策略 & 15 & 0.341 & 1.429 & 0.148 \\
\cline { 2 - 6 } & 交互 & 15 & 2.052 & 1.927 & 0.029 \\
\hline \multirow{2}{*}{ 误差 } & 互动类型 & 102 & 0.243 & & \\
\cline { 2 - 6 } & 互动策略 & 102 & 0.239 & & \\
\cline { 2 - 6 } & 交互 & 102 & 1.065 & & \\
\hline
\end{tabular}

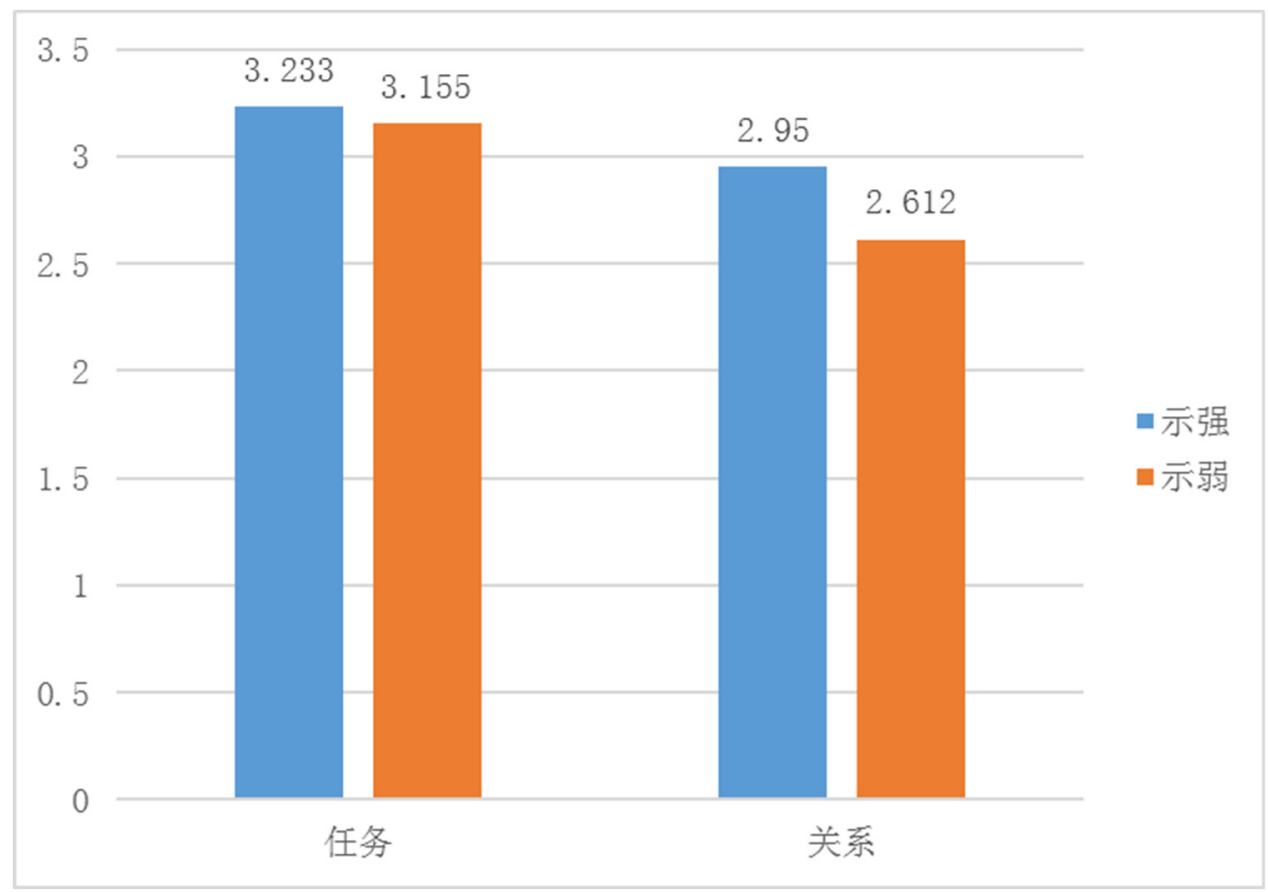

图 2 游戏直播中不同互动类型、互动策略对情感能量的影响 
然后，验证秀场直播的假设。可以看出秀场直播中不论是任务还是关系导向型互动， 都是采取示弱策略比采取示强策略效果更好。不论是采取示强还是示弱策略, 关系导向型互 动都比任务导向型的互动效果要好。结果支持假设 $\mathrm{H} 1 \mathrm{~d}$,但是不支持假设 $\mathrm{H} 1 \mathrm{c}$ 。即：在秀场直 播中, 不管是怎样的互动类型, 示弱策略都优于示强策略, 不论怎样的互动策略, 都是关系 导向型互动优于任务导向型互动。

再次验证不同直播类型下，不同的互动类型时，主播互动策略对主播认同的影响是否存 在差异。结果和情感能量的大体分析结果一致, 受篇幅所限, 就不一一列出表和图了。结果 游戏直播中支持 $\mathrm{H} 2 \mathrm{a}$, 但不支持 $\mathrm{H} 2 \mathrm{~b}$,秀场直播中支持 $\mathrm{H} 2 \mathrm{~d}$, 但不支持 $\mathrm{H} 2 \mathrm{c}$ 。

表 2 秀场直播中不同互动类型、互动策略对情感能量的影响

\begin{tabular}{|c|l|l|l|l|l|}
\hline & 因变量 & 自由度 & 均方 & F统计量 & P值 \\
\hline \multirow{5}{*}{ 情感能量 } & 互动类型 & 14 & 0.987 & 5.779 & 0 \\
\cline { 2 - 7 } & 互动策略 & 14 & 0.378 & 1.591 & 0.09 \\
\cline { 2 - 7 } & 交互 & 14 & 4.38 & 5.22 & 0 \\
\hline \multirow{3}{*}{ 误差 } & 互动类型 & 127 & 0.171 & & \\
\cline { 2 - 7 } & 互动策略 & 127 & 0.238 & & \\
\cline { 2 - 6 } & 交互 & 127 & 0.839 & & \\
\hline
\end{tabular}

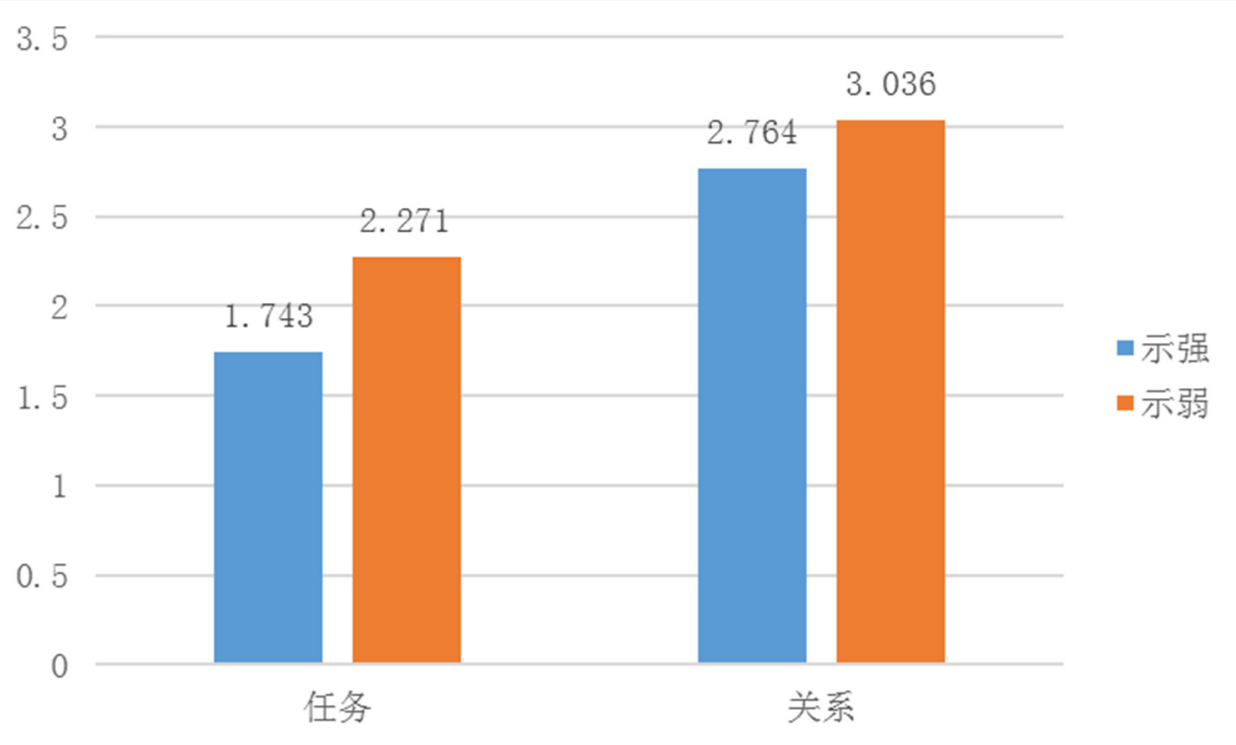

图 3 秀场直播中不同互动类型、互动策略对情感能量的影响

最后验证情感能量、主播认同和打赏意愿三者之间的关系。三个变量之间相关关系的显 著性 P 值均小于 0.05 , 但是不同变量之间相关系数的大小有一定差异其中, 情感能量与主播 认同的相关系数为 0.761 , 情感能量与打赏意愿的相关系数为 0.595 , 主播认同与打赏意愿相 关系数为 0.699 。说明三个因变量之间有着较为明显的正向关系。与假设 H3、H4、H5 基本 一致。 
表 3 情感能量、主播认同和打赏意愿的相关分析

\begin{tabular}{|ll|r|r|r|}
\hline 变量 & 指标 & 打赏意愿 & 情感能量 & 主播认同 \\
\hline 打赏意愿 & Pearson 相关性 & 1 & $.595^{* *}$ & $.699^{* *}$ \\
& 显著性 (双侧) & & .000 & .000 \\
情感能量 & Pearson 相关性 & $.595^{* *}$ & 1 & $.761^{* * *}$ \\
& 显著性 (双侧) & .000 & & .000 \\
主播认同 & Pearson 相关性 & $.699^{* *}$ & $.761^{* *}$ & 1 \\
& 显著性 (双侧) & .000 & .000 & \\
\hline
\end{tabular}

\section{2 理论贡献}

本研究丰富和深化了网络直播的打赏机制的理论研究，对后续相关研究也有一定的借鉴 意义和推动作用, 理论贡献主要如下。

(1) 对网络直播进行实证研究, 丰富了网络直播的研究领域和内容。由于网络直播属于 近年来才逐渐开始走红的事物, 国内外对于网络直播打赏意愿的研宄都相对较少, 实证研究 也并不多见。因此, 本研究进一步丰富了网络直播的研究内容, 拓展了网络直播的研究领域。

(2) 本研究引入了社会学中的互动仪式链理论, 其核心是情感能量, 丰富了互动仪式链 研究的内容, 推动将互动仪式链理论应用于营销环境之中; 另一方面, 将劣势者效应与社会 认同理论相结合来诠释互动策略对直播受众信息处理过程和结果的影响机制。研究表明, 互 动仪式链理论和社会认同理论对受众打赏意愿机制具有较大的解释力, 对后续相关研究有一 定的借鉴价值。

（3）从认知和情感角度分析受众打赏意愿, 深化了网络直播的互动响应机制研究。在网 络直播迅速发展的时代, 至少在目前网络直播的盈利模式主要还是靠受众的打赏, 因此如何 激发受众的打赏意愿和行为已成为网络直播的重要研究课题。本研究将研究重点聚焦于受众 对网络主播的打赏意愿，并在 $\mathrm{Hu} \mathrm{M}$ 等（2017）、Sjöblom\&Hamari（2017）、Hilvert-Bruce Z 等 (2018) 等学者的研究基础上, 从认知和情感两个角度出发, 较深入地分析了情感能量、 主播认同与受众打赏意愿的作用关系, 较全面地厘清了不同直播类型、互动类型-策略对受众 打赏意愿的具体影响机制，推动了网络直播的互动响应机制的研究不断深化。

\section{3 管理启示}

(1) 本文对网络直播的发展间接性提出了建议。目前, 经营问题, 直播人数的造假问题, 直播内容涉黄违法问题等等都困扰着各大网络直播平台进一步的发展。本文从主播互动的角 度对网络直播的良性发展提出了间接性的建议。主播直播以内容为主, 但是也不能忽视直播 技巧, 要能让受众对你的内容感兴趣, 因此直播平台主播应该充分利用互动类型和互动策略 的巧妙融合, 使其既能给受众带来生动、直观、感性的娱乐体验, 同时还能吸引那些潜在客 户对主播打赏, 增加平台和主播的收入。

(2) 合理运用互动类型和互动策略。在不同的主播类型的结果不一样, 本文游戏和秀场 直播在互动类型和互动策略的选择上是有区别的, 在游戏直播中, 主播应该侧重任务导向的 互动和示强的策略, 尽可能充分发挥主播的游戏技术水平来打动受众, 尽可能表现出自己厉 害的一面; 在秀场直播中, 主播应该侧重关系导向的互动和示弱的策略, 尽可能和受众保持 情感互动, 安抚受众的情绪, 在受众面前尽可能示弱, 博取受众的关注和怜爱。当然不论游 戏直播和秀场直播, 如游戏直播, 不能忽视关系导向的互动, 或者是示弱的互动策略, 在必 要的时候也是需要交叉使用的，同理秀场直播也是一样的。 


\section{4 局限性与未来研究展望}

(1) 针对样本的收集

受制于客观条件, 本课题仍然采用问卷形式, 由受访者自行填写, 不可避免地会出现主 观性过强的问题。

（2）缺少专门的模型和方法

因为直播真正产业化和规模化的时间尚短，目前也只是成了众多娱乐方式中的一种，因 此也没有引起学术界的过多关注。

（3）直播涉及的因素过多，难以精确量化

直播看似只是主播与观众的互动, 实际上影响因素众多, 又难以用数据去量化, 比如从 主播方面看, 他 (她) 的学历、见识、风格、经历、外貌、口头禅、小动作、表演水平、互 动水平等; 从直播的内容来看, 游戏的类型、游戏的阶段、游戏的画面、游戏的流畅性、游 戏运营策略等; 从直播的用户来看, 用户的年龄、学历、文化水平、职业、地区、消费能力 等都可能对直播的效果与体验造成一定影响，最终会导致模型非常复杂。

\section{References}

[1] Baker J O. Social Sources of the Spirit: Connecting Rational Choice and Interactive Ritual Theories in the Study of Religion[J]. Sociology of Religion, 2010, 71(2):432-456.

[2] Collins R. Interaction Ritual Chains[J]. Contemporary Sociology, 2009, 38(2):191-192.

[3] Dodds W B, Monroe K B, Grewal D. Effects of price, brand, and store information on buyers' product evaluations.[J]. Journal of Marketing Research, 1991, 28(3):307-319.

[4] $\mathrm{Hu} \mathrm{M}$, Zhang M, Wang Y. Why do audiences choose to keep watching on live video streaming platforms? An explanation of dual identification framework[J]. Computers in Human Behavior, 2017,75 .

[5] Köhler C F, Rohm A J, Ruyter K D, et al. Return on Interactivity: The Impact of Online Agents on Newcomer Adjustment[J]. Journal of Marketing, 2011, 75(2):93-108.

[6] Kelman H C. Process of Opinion Change[J]. Public Opinion Quarterly, 1961, 25(1):57-78.

[7] Paharia N, Keinan A, Avery J, et al. The Underdog Effect: The Marketing of Disadvantage and Determination through Brand Biography[J]. Journal of Consumer Research, 2011, 37(5):775-790.

[8] Shirom A. FEELING VIGOROUS AT WORK? THE CONSTRUCT OF VIGOR AND THE STUDY OF POSITIVE AFFECT IN ORGANIZATIONS[J]. Research in Occupational Stress \& Well Being, 2003, 3(6):135-164.

[9] Shamir B, Zakay E, Breinin E, et al. Correlates of charismatic leader behavior in military units: Subordinates' attitudes, unit characteristics, and superiors' appraisals of leader performance.[J]. Academy of Management Journal, 1998, 41(4):387-409. 